\title{
EN TORNO A los DERECHOS ON-LINE DE LOS TRABAJADORES Y
} DE SUS ORGANIZADORES

\author{
Miguel C. Rodriguez-Piñero Royo* \\ Roberto Fernández Villarino**
}

\section{RESUMEN:}

Este artículo analiza el impacto de las tecnologias de la infocomunicación en las relaciones laborales, particularmente en lo que se refiere a los derechos de los trabajadores $y$ de sus representantes en la empresa. Se parte para ello del concepto de derecho on-line, elaborado por las organizaciones sindicales europeas, y se estudia el nivel actual de garantia en el Derecho laboral español. El análisis se bace tanto en la legislación vigente como en la práctica jurisprudencial y en la negociación colectiva, concluyendo con las propuestas de reforma que se estân manejando en nuestro paús.

\section{Palabras Clave:}

Tecnologias de la infocomunicación. Derechos on-line. Representantes de los trabajadores. Derecho a la intimidad. Control empresarial. Derechos de acceso.

\section{ABstract:}

This paper analyses the impact of new technologies on labour relations, particularly regarding those rights of workers and their representatives at the enterprise. The starting point is the definition of on-line rights, a concept which bas been elaborated by European unions as a basis for the debate. The current level of recognition and protection of such rights under Spanish labour law is analyzed. This analysis is performed at three levels: labour legislation currently in force; case law; and collective bargaining. The paper ends with a study of the proposals to reform Spanish Labour Law to adapt it to these rights.

- Catedrático de Derecho del Trabajo y de la SS. Universidad de Huelva - mrodriguez@uhu.es

- Profesor Asociado de Derecho del Trabajo y de la SS. Universidad de Huelva - roberto@uhu.es 
KEY WORDS:

Information and communication technologl'. On-line rights. Workers' representatives. Privacy. Management control rights. Access rights.

\section{PRESENTACIÓN}

La conexión entre el Derecho del Trabajo, el sistema productivo y la tecnología que éste utiliza es bien conocida, y no merece ulteriores comentarios. Esta rama del ordenamiento jurídico se diseña y elabora de acuerdo con un modelo productivo, y pensando en la utilización de unas tecnologías determinadas, de tal modo que los cambios en éstas y en aquél ponen en cuestión su justificación y su eficacia reguladora, forzándole a actualizaciones constantes. Más aún. en la explicación más generalizada de los origenes históricos del Derecho del Trabajo éste se presenta como la respuesta del sistema jurídico a un fenómeno, la Revolución Industrial. que es a su vez consecuencia de cambios en los sistemas de producción motivados por la implantación de mejoras técnicas. Muchas de las crisis de crecimiento y de adaptación de la normativa laboral se presentan, además, como la consecuencia de cambios en la producción producidos por ulteriores novedades técnicas. Ramas enteras del Derecho del Trabajo, como el Derecho de la Prevención de Riesgos Laborales, presentan un condicionante tecnológico aún mayor, tanto en los riesgos que pretende evitar como en los instrumentos que utiliza para ello.

En este contexto hablar de "tecnologia" en el ámbito de los estudios laborales siempre resulta justificado. Más peligroso resulta hablar, por el contrario, de "nuevas tecnologías", porque la novedad cuando se habla de éstas es un calificativo siempre discutible y perecedero. Particularmente porque cuando los juristas nos planteamos analizar el impacto de una nueva tecnología, ésta se encuentra por lo general tan extendida en el sistema productivo que difícilmente soporta este calificativo. Sólo así puede considerarse un problema social, y sólo así puede provocar una reacción del ordenamiento jurídico..

La generalización de las tecnologías de la infocomunicación (TIC) en el ámbito productivo ha hecho surgir una serie de cuestiones que van más allá de la mera adaptación a los cambios tecnológicos a que el Derecho del Trabajo tiene que enfrentarse continuamente. Los cambios de todo tipo que suponen en prácticamente cada ámbito de las relaciones laborales constituyen sin lugar a dudas uno de los núcleos principales de atención para el jurista del trabajo en el inicio del milenio.

Aunque los efectos de estas nuevas tecnologias son múltiples y multidireccionales, se puede sugerir un intento de sistematización en algunas pocas grandes líneas: 
- en primer lugar, cambios en la forma de trabajar producidos no sólo por la disponibilidad de estos nuevos instrumentos tecnológicos. sino también por la reorganización de las formas de producción que éstos han provocado. Podemos encontrar incluso formas de empleo de nuevo cuño, distintas a las tradicionales, lo que incluye desde luego al trabajo remoto o teletrabajo, en la terminología al uso. A su vez, esta nueva forma cle trabajar genera nuevos riesgos en el trabajo, relacionados con los instrumentos y las energías con que se opera, y con la interacción de éstos con el trabajador. Riesgos por pantallas de ordenador. por posturas en el trabajo. por sobrecarga laboral. por sólo citar algunos, están a la orden del día en las empresas que utilizan de forma masiva estas tecnologías.

- En segundo lugar. nuevas posibilidades de control empresarial sobre los trabajadores, en aspectos que no se limitan ya a su productividad y conducta en la empresa, sino que alcanzan a su comportamiento en general. a su actividad extralaboral y a su vida privada.

- En tercer lugar, nuevas posibilidades de actuación para las organizaciones de trabajadores; los medios tecnológicos disponibles abren nuevas vías para la acción sindical en sus diversas manifestaciones: organización de los trabajadores, relaciones internas del sindicato. presencia sindical en la empresa, convocatoria de acciones colectivas, difusión de todo tipo de informaciones, etc....

- En cuarto lugar. una nueva posición del trabajador en el mercado de trabajo, pues ésta dependerá principalmente de sus habilidades en relación con estas tecnologías. Además, el ritmo de cambios ténológicos acelerado le somete al riesgo de perder su empleabilidad como consecuencia del desfase de sus conocimientos, y la obsolescencia de su formación. La formación profesional adquiere una nueva dimensión, y no se limitará a los períodos previos al inicio de su carrera profesional. Esta carrera profesional se verá también afectada, sustituyéndose el empleo permanente en una empresa por una sucesión de distintos empleos, seguramente también de distintos trabajos. intercalados con períodos de formación, entrando y saliendo con frecuencia del mercado de trabajo.

- En quinto lugar, un difuminado de las fronteras entre la actividad profesional y la vida privada del trabajador, al que se le coloca en una situación de localización permanente, se le invade el hogar con medios técnicos de la empresa, y se le alarga la jornada laboral.

Muchos de los temas de actualidad del Derecho del Trabajo, en el nuestro como en otros países, giran en torno a estas cuestiones. La protección de los datos personales del trabajador, la privacidad de sus comunicaciones, la monitorización de sus actividades, la utilización de los nuevos canales de comunicación para la actividad sindical, la propiedad intelectual de los resultados de la actividad de los trabajadores, la 
protección contra los riesgos profesionales derivados de estas tecnologías, la distinción entre lo profesional y lo personal en el tiempo de trabajo, los efectos de la propiedad de los-medios de comunicación sobre la delimitación de su uso y su función..., ocupan hoy la atención de los operadores jurídicos del mercado de trabajo y centran las prioridades de los actores del sistema de relaciones laborales. En algunas ocasiones se trata tan sólo de cuestiones tradicionales que adoptan nuevas formas como consecuencia de los cambios tecnológicos experimentados, o que incrementan su intensidad; en ocasiones, por el contrario, éstos hacen aparecer problemas completamente nuevos. En uno y otro caso se plantea un auténtico desafío para una regulación laboral surgida en un contexto tecnológico y productivo completamente diferente. Sólo que en algunos casos se dispondrá de respuestas normativas expresas, en ocasiones inadecuadas o insuficientes, y en otros se carecerá por completo de ellas.

\section{LOS DERECHOS ON-LINE COMO CATEGORÍA JURÍDICA}

A lo largo de su historia el Derecho del Trabajo ha sido capaz de generar un cuadro de regulación de las relaciones laborales que ha respondido, entre otras, a dos consideraciones: una voluntad de garantizar la dignidad del trabajador en su relación de empleo y en su vida social; y la búsqueda de un modelo democrático de relaciones laborales, en el que la empresa se constituya en un ámbito de participación que supere la lógica simplista de la propiedad de los medios de producción. Para poner en práctica uno y otro objetivo el ordenamiento jurídico ha desarrollado un conjunto de derechos, tanto individuales como colectivos, reconocidos y garantizados en el plano normativo, y que configuran un determinado modelo de civilización en el mercado de trabajo y en el sistema productivo en general.

Desde el punto de vista del Derecho del Trabajo, se plantean nuevas posibilidades de lesión de los derechos legales de los trabajadores y sus organizaciones, sobre todo los relacionados con la intimidad; y una pérdida de efectividad de otros derechos tradicionales de actuación colectiva, vinculados principalmente a la comunicación entre éstas y aquéllos. Con ello la eficacia del ordenamiento laboral en el logro de sus objetivos prioritarios puede reducirse considerablemente. Para responder a estos desafíos la respuesta del ordenamiento jurídico debe ser la actualización del conjunto de derechos que se reconocen a los trabajadores y organizaciones, adecuando los ya existentes a los nuevos condicionantes tecnológicos que puedan cuestionar su sentido y su eficacia, y diseñando aquellos otros de nueva creación que resulten necesarios para mantener lós objetivos de dignidad del trabajador y de democratización de la empresa. 
Como forma de clasificar y visualizar los derechos vinculados a las nuevas tecnologías en la empresa se ha acunado la categoría de "derechos online". Es ésta una expresión que empezó a generalizarse en el ámbito sindical en la segunda mitad de la década de los noventa para hacer referencia a una nueva generación de derechos, relacionados con la utilización de las nuevas tecnologías, en particular de las que utilizan el soporte de Internet. Esta categoría ha sido ya aceptada por la doctrina laboralista, y hoy podemos considerarla consolidada. Tiene la virtud de darle nombre y tratamiento integrado a una pluralidad de reivindicaciones muy distintas, que comparten sin embargo el elemento común de vincularse en su ejercicio o en sus efectos a las TIC.

A pesar de ser un concepto todavia reciente', existe ya un cierto acuerdo sobre su contenido y límites. En las páginas que siguen nos couparemos de desarrollar una primera aproximación a éstos.

Desde un punto de vista tecnológico la expresión on-line, en el uso que se le da en el lenguaje común, se refiere a la comunicación uni- o bidireccional a través de diversos canales -cable, línea telefónica-, en formato digital. Aplicados a los derechos, y hablando de "derechos on-line", la conclusión es que esta categoría incluye a derechos relacionados con tan sólo algunas de las manifestaciones de las TIC, pero no a todas ellas. El uso de la expresión "on-line", en efecto, parece indicar servicios o actividades desarrolladas a través del soporte de la red, Internet u otras, en los que se produce una transmisión de datos en soporte electromagnético, excluyendo otras manifestaciones tecnológicas ${ }^{2}$.

Aún así, en los estudios que comienzan a generalizarse sobre esta categoría suelen aparecer derechos en los cuales la interconexión no se produce necesariamente, como puede ser la protección de los datos personales del trabajador recogidos y gestionados de forma informatizada. Es evidente que muchas de las cuestiones suscitadas por el uso de medios tecnológicos en las relaciones laborales se producen en medios en los que no se produce esta conexión en línea; pensemos, por ejemplo, en trabajadores que manejan or-

1. Un primer análisis de esta categoria desde el Derecho español en F. BARBA RAMOS \& M. RODRIGUEZ-PIÑERO ROYO, "Alternativas de regulación de los derechos on-line en el ordenamiento laboral español", Derecho y Conocimiento, n. 1, 20002.

2. Quedarian excluidas, por tanto, las conexiones telefónicas tradicionales, el fax, o los teléfonos móviles, siendo todos éstos encuadrables dentro del concepto general de TIC. 
denadores independientes entre sí, en teletrabajadores sin conexión con el ordenador de la empresa, etc... Seguramente sería más correcto, por ello, hablar de "derechos informáticos" de los trabajadores y sus organizaciones, una nueva manifestación del llamado "Derecho informático"

Desde un punto de vista subjetivo titulares de los derechos on-line son los trabajadores y sus representantes, tanto unitarios como sindicales. Técnicamente son derechos de titularidad individual y colectiva, encuadrables dentro de las distintas manifestaciones de la libertad sindical y de los derechos de representación de los trabajadores en la empresa. Lo que excluye en a otros sujetos de las relaciones laborales, como las asociaciones empresariales, la administración laboral, los demandantes de empleo o los beneficiarios de prestaciones del sistema de seguridad social. Ello es así porque estos derechos se plantean sobre todo frente al empresario, como titular de los medios tecnológicos sobre los que se ejercitan. Se trata, en suma, de reconocer el derecho a acceder a estos medios por parte de trabajadores y representantes de éstos, y de ordenar las condiciones de este acceso ${ }^{3}$.

Como muestra de la delimitación de los derechos on-line podemos utilizar el Código de Conducta elaborado por la Union Network International (en adelante UNI), en el contexto de una campaña iniciada en 1998 a nivel mundial para apoyar su reconocimiento. Esta organización centra sus actividades en la red, por lo que su concepto de derecho on-line se ciñe a este medio de comunicación, siendo por ello relativamente estricto. Para esta organización, los derechos que deben reconocerse a los trabajadores y a sus organizaciones en el acceso a la red son los siguientes:

- el derecho para los empleados, los sindicatos y los comités de empresa de tener libre acceso a los sistemas de correo electrónico de las empresas con el fin de recibir información y comunicarse.

- el derecho de los empleados a tener el libre acceso a Internet (y a las redes intranet de las empresas) para poder acceder a las páginas web de su sinidicato y a otras informaciones relacionadas con sus derechos como trabajador.

3. La consecuencia es que se excluyen otros derechos de otros sujetos que, en la práctica, pueden tener un nivel de reconocimiento mucho mayor en estos momentos: por ejemplo, el derecho de los demandantes de empleo de acceder a las bases de datos de las oficinas de colocación, la garantía de la confidencialidad de los datos que se disponen de ellos, la comunicación electrónica con las entidades gestoras de la Seguridad Social -que es hoy la regla a partir del programà R:EA-, la protección de sus datos personales, etc... Estos derechos estanian garantizàdos al día de hoy conla legiślación general sobre protección de datos y la normativa administrativa correspondiente. 
- ninguna vigilancia electrónica secreta de los mensajes de correo electrónico o páginas web visitadas por los empleados.

Como se ve los derechos on-line, en este sentido restringido o limitado, giran en torno a dos grandes principios: el derecho a la utilización de las TIC basadas en la red para la comunicación entre los trabajadores y sus representantes, de un lado; y la garantía de la privacidad en estas comunicaciones, de otro. En términos informáticos, hablamos de access y de privacy, de acceso y de intimidad; $O$, dicho en otras palabras, de un acceso con todas las garantías, de un acceso pleno a la red para las comunicaciones sindicales, lo que significa no sólo que éste sea permitido, sino que se dispongan de los medios técnicos necesarios para hacerlo posible -conexiones y estaciones de trabajo-, de la información suficiente-direcciones de correo, códigos de acceso-, y que ésta se realice con la suficiente protección de los datos relativos a todos los sujetos implicados. En resumen, que se haga posible la acción sindical a través de la red de la empresa, en condiciones similares a cómo ésta viene siendo desarrollado por los cauces tradicionales.

No se mencionan, por el contrario, otros posibles derechos igualmente relacionados con la red, como podría ser el derecho a disponer de un tablón de anuncios electrónico en la Intranet de la empresa, algo muy frecuente ya en muchas empresas y administraciones públicas; el derecho a obtener información sobre la utilización de la red; el derecho a participar en el diseño y gestión de la red corporativa; la gestión participada del portal de empleados de la empresa -comunicación B-to-E, etc... Tampoco quedan claramente recogidos otros derechos vinculados a la acción sindical, como el referéndum electrónico, la teleasamblea o asamblea a través de videoconferencias, las medidas de presión colectiva... aunque se podría pensar que todas ellas estarían incluidas dentro de las facilidades de comunicación a través de la red que los derechos on-line suponen.

En términos materiales estos derechos on-line no suponen necesariamente una ampliación de los derechos de los que legalmente disfrutan ya trabajadores, representaciones unitarias y sindicatos. Se trata, más bien, de una adaptación de éstos a un nuevo entorno tecnológico, que permita cumplir sus objetiyos originales con un soporte material nuevo. Incluso en la terminología sindical se mantienen las denominaciones tradicionales de los instrumentos de acción sindical, con algún retoque para indicar el cambio de soporte tecnológico: teleasamblea, telerreferendum, tablón de anuncios sindical... Todas estas expresiones evocan formas de comunicación tradicionales, adaptadas a los nuevos tiempos. En general puede afirmarse que existe un cierto interés de los defensores de estos derechos on-line por dejar claro que no se trata de reconocer prerrogativas de nuevo cuño, sino de mantener la vigencia de las preexistentes. 
Todo lo más se reconoce el derecho a utilizar las infraestructuras informáticas y telemáticas de la empresa para usos representativos, distintos de los estrictamente relacionados con la producción; pero con un coste para la empresa en principio muy pequeño. En muchos casos, además, es previsible que se opere una transacción o sustitución de unos derechos de representación y acción sindical tradicionales por los nuevos, por lo que no se acumularian éstos con aquellos. Así ocurrirá, claramente, con el tablón de anuncios electrónico, que sustituirá al físico; las videoconferencias desplazarán a las asambleas presenciales; el correo electrónico, al reparto de materiales informativos.

- Junto a estos derechos de acceso y privacidad la propia UNI ha manejado otros derechos que podrían igualmente incluirse en esta categoría general de derechos on-line; si bien lo ha hecho tan sólo en determinados documentos, sin incluirlos expresamente dentro de las prioridades de su campaña para su reconocimiento normativo. Así, se ha hablado del derecho a la formación en materia de tecnologías relacionadas con la red, al efecto de evitar la exclusión informática de los trabajadores; entendiendo por ésta la pérdida de condiciones profesionales y de sociabilidad que supone la incapacidad de utilizar las herramientas on-line en un contexto en el que un porcentaje cada vez mayor de las comunicaciones se produce por esta vía. La falta de conocimientos informáticos puede traer consigo un aislamiento del trabajador, su marginación en el mercado de trabajo, la pérdida de expectativas profesionales... En este contexto, el derecho de acceso a la red para los trabajadores no debe entenderse limitado a una función de comunicación sindical, sino en un sentido mucho más amplio, como mecanismo para evitar este peligro de marginación. La formación y el soporte técnico que lo hagan posible deben ser valorados desde este punto de vista.

En conclusión, la categoría de "derechos on-line" es una relativamente limita$\mathrm{da}$, que no incluye ni todos los posibles derechos relacionados con las nuevas tecnologías, ni a todas éstas, ni a todos los posibles sujetos interesados en su reconocimiento. Aún así resulta una formulación sugerente, porque encuentra una base común para tratar de manera conjunta una pluralidad de situaciones que, con la dogmática jurídica tradicional, se englobarían entre los contenidos de distintos derechos fundamentales; y este tratamiento unitario tiene sentido desde el momento en que la base tecnológica es común. Por ello, y por que se trata de una categoría aceptada por los interlocutores sociales como base para sus reivindicaciones, la utilizaremos en este estudio.

Finalmente, resulta conveniente señalar cómo la doctrina científica sobre løs derechos humanos habla ya de una cuarta generación de éstos, que incluiría derechos relacionados con las nuevas tecnologías y con el medio ambiente. Esta categoría incluiría claramente a los derechos on-line, que serían, técnicamente hablando, derechos fundamentales de cuarta generación. 


\section{LOS DERECHOS ON-LINE EN EL ORDENAMIENTO LABORAL ESPAÑOL:} ESTADO DE LA CUESTIÓN

\subsection{LOS DERECHOS ON-LINE EN ESPANA: VALORACIÓN GENERAL.}

En el momento actual del ordenamiento del trabajo español estos derechos no están garantizados ${ }^{4}$. El análisis de los materiales normativos disponibles confirma esta opinión: ni en los textos legislativos y reglamentarios que constituyen nuestro Derecho del Trabajo, ni en la negociación colectiva encontramos un tratamiento adecuado de estas cuestiones, tanto de los derechos on-line estrictamente hablando, como de otros muchos problemas planteados por las TIC en las relaciones laborales.

No olvidemos que el Derecho del Trabajo español responde a un contexto tecnológico y productivo muy diferente al que la sociedad del conocimiento nos impone. Los derechos y las garantías reconocidos a los trabajadores y a sus representantes se disenaron de acuerdo con las necesidades y los medios técnicos disponibles en un momento histórico determinado. La revolución tecnológica experimentada en los últimos años ha hecho peligrar su eficacia, y con ello se ha reducido el grado de penetración del Derecho del Trabajo, su capacidad para ordenar realmente el mercado de trabajo. En un ordenamiento constantemente preocupado por mantener sus niveles de efectividad, ante los intentos continuos de huida y de inaplicación, un fenómeno de este tipo no deja de ser preocupante.

Los problemas de obsolescencia normativa suelen solucionarse por la labor interpretativa de la jurisprudencia. En este caso, sin embargo, no parece que los tribunales de trabajo hayan sido capaces de superar las limitaciones del Derecho vigente ${ }^{5}$; y ello por una serie de razones: la primera, que éste resulta particularmente inadecuado, por lo preciso de su redacción en muchos casos, como veremos. La segunda es que los

4. Un estudio de conjunto de estos problemas en M. RODRIGLEZ-PIÑERO ROYO, "Employment and privacy: Electronic interaction in the workplace in Spain", Bulletin of Comparative Labour Relations, 2002; F. BARBA RAMOS \& M. RODRÍGUEZ-PIÑERO ROYO, "Alternativas de regulación de los derechos on-line en el ordenamiento laboral español", Derecbo y Conocimiento, $\mathrm{n}^{\circ} 1,2002$.

5. Para un análisis de esta jurisprudencia, entre otros, J.I. GARCIA NINET, "Sobre el uso y abuso del teléfono, del fax, del ordenador y del correo electrónico de la empresa para fines particulares en lugar y tiempo de trabajo. Datos para una reflexión en torno a las nuevas tecnologías", Tribuna Social; n. 127, 2001, pg.5 sigts.; J. LUJAN ALCARAZ. "Uso y control en la empresa de los medios informáticos de comunicación", Aranzadi Social, n.2, 2001. pg.11 sigts; J.J. DE VAL ARNAL, "El correo electrónico en el trabajo y el derecho a la intimidad del trabajador", Aranzadi Social, n.17, 2000, pg.25 sigts; A.V. SEMPERE NAVARRO \& C. SAN MARTÍN MAZZUCONI, "Nuevas tecnologías y relación de trabajo", Aranzadi, Pamplona, 2002; M. RODRÍGUEZ-PIN̄ERO ROYO, R. FERNÁNDEZ VILLARINO, F. BARBA RAMOS \& J.L. LÁZARO SÁNCHEZ, "Nuevas tecnologias y Derecho del Trabajo: estado de la cuestión en España", en J. CASTIÑEIRA FERNÁNDEZ; "Jornadas Andaluzas de Derecho del Trabajo". Monografias de Temas Laborales, Sevilla, en prensa. 
tribunales contaban ya con ciertas construcciones relativas a medios de comunicación, pero elaboradas en momentos anteriores, cuando la tecnología era muy diferente; la aplicación de los criterios jurisprudenciales sobre el uso privado del teléfono al correo electrónico, por ejemplo, no ha dado siempre buenos resultados. Aunque quizás la principal razón sea que los jueces de trabajo no han sido particularmente imaginativos en la aplicación de las bases normativas de las que disponían para dar respuesta a algunos de lọs problemas planteados por las TIC.

Las alternativas que quedan para acompasar el Derecho español a las nuevas situaciones son numerosas: reformas legislativas, por supuesto, y de ello se ha hablado, como veremos. Pero también podría servir una interpretación evolutiva del Derecho vigente, que superara la literalidad precisa de las normas vigentes para permitir acoplarlas a las nuevas TIC. Como por el momento ni lo uno ni lo otro se ha producido, ha sido la negociación colectiva la que se ha ocupado de afrontar algunas de estas cuestiones. Ésta es la vía que parece más prometedora, ante la aceptación por parte de los interlocutores sociales de la necesidad de ofrecer a las empresas un marco adecuado para el uso de las TIC. El proceso de introducción de estos temas en los convenios colectivos españoles ya se ha iniciado, aunque todavía se encuentra en un momento inicial, estando tratada en un número pequeño de convenios ${ }^{6}$.

La otra alternativa de regulación, que es la que en la práctica más se utiliza, es la actuación unilateral de la empresa, mediante la elaboración de normativas internas sobre la utilización de las TIC que empiezan a ser frecuentes en las grandes empresas. Ante las limitaciones de esta solución están apareciendo otros tipos de documentos que se ocupan de estas cuestiones, como son las directrices o recomendaciones de órganos especializados, los códigos de buenas prácticas elaborados por organizaciones privadas, las plataformas negociales, etc...

\subsection{Protección de la privacidad del trabajador y uso privado de las tiC}

Uno de los temas recurrentes en España es el de la protección de la privacidad del trabajador en su utilización de los medios TIC en su entorno de trabajo. El tratamiento de esta cuestión, de por sí trascendental, se complica al aparecer ésta por lo general vinculado con otra, igualmente compleja: la utilización de estos medios con fines privados. El supuesto tipo es el control por la empresa del uso de los medios de comunica-

6. Según el Informe de la CEOE sobre la negociación colectiva en España en el año 2001: "(...) Es, igualmènte, el caso de las nuevas tecnologías. Sólo el $4 \%$ de los convenios tegula algún aspecto vinculado a esta cuestión, y apenas lo hacen el $6 \%$ de los convenios de empresá El aso del correo electrónico, por el que CEOE se interesa particularmente, se regula en el $5 \%$ de los eonivenios en lo que respecta a su uso por los representantes de los trabajadores. Un porcentaje stimilar (pero que alcanza el $12 \%$ de los convenios de empresa) regula como falta el uso inadecuado de aquel instrumento tecnológico por parte de los trabajadores." 
ción puestos a disposición del trabajador, para comprobar si éste se limita a finalidades relacionadas con el trabajo, o si por el contrario incluye comunicaciones privadas de éste -primer problema-; para poder determinar la naturaleza de esta utilización debe procederse a un análisis de las comunicaciones efectuadas por éste, lo que puede vulnerar si intimidad, o la privacidad de las comunicaciones -segundo problema-. No es que el Derecho español desconozca las cuestiones relacionadas con la intimidad del trabajador. De hecho, son los preceptos estatutarios dedicados a proteger ésta, los artículo 18 y 20 , los que son utilizados por los tribunales espanolas para afrontar estas cuestiones. Tampoco son nuevos los problemas provocados por una utilización de los medios de la empresa para fines privados. Sólo que las soluciones tradicionales no resultan adecuadas por estar diseñadas para un entorno tecnológico distinto, y para problemas diferentes a los que las TIC están planteando.

Quizás el caso que mejor refleje este tipo de problemas sea el asunto conocido como "caso Deutsche Bank", que supone una muestra fiel de los distintos aspectos jurídicos implicados: un trabajador fue despedido por usar el ordenador de la empresa para enviar comunicaciones privadas, lo que la empresa conoció por su registro de éste. En vía laboral el asunto concluyó ante la Sala de lo Social del Tribunal Superior de Justicia de Cataluña, que entendió legítimo tanto el registro como el despido. En vía penal el trabajador denunció a la empresa, por violación del secreto de las telecomunicaciones, admitiéndose a trámite su denuncia. Ni una ni otra vía llegaron a culminarse del todo, puesto que la Sala de lo Social del Tribunal Supremo inadmitió el recurso de casación para la unificación de doctrina presentado por el trabajador; y éste retiró su denuncia en vía penal tras haber llegado a un acuerdo económico con la empresa. En todo caso, de la sentencia del TSJ se desprende una doctrina que en líneas generales coincide con la interpretación judicial mayoritaria en nuestro país. Intentaremos delimitar esta jurisprudencia identificado las cuestiones esenciales planteadas.

\subsubsection{Tratamiento en la jurisprudencia laboral}

Una primera cuestión recurrente en la doctrina jurisprudencial es la relativa a la aplicación o no del artículo $18 \mathrm{ET}$ a los controles y registros desarrollados por la empresa en el ordenador, conexiones y cuentas de correo utilizados por el trabajador. Este precepto, de aplicación en principio a los registros en la taquilla y objetos personales del trabajador, ha sido considerado aplicable por la sentencia del STSJ de Andalucía de 25 de febrero de 2000: "(..) El artículo 18 del E.T. precepto al amparo del cual la empresa realizó el registro informático, autoriza la realización de registros sobre la persona del trabajador, en sus taquillas y efectos particulares (...) Por lo tanto dicbo precepto autoriza el registro en la terminal de ordenador que utiliza el trabajador, pues el ordenador a estos efectos puede asimilarse a la taquilla, ya que no podemos olvidar que dicho ordenador, es un instrumento de trabajo propiedad de la empresa y que no debe ser utilizado 
para otros fines distintos y diferentes que la realización de la actividad laboral, por lo que no cabe bablar de documentos personales incorporados al mismo". A la misma conclusión llega el Tribunal Constitucional en sentencia de 10 de julio de 2000: "efectuar registros en los terminales de ordenador de los trabajadores no es un derecho absoluto $e$ incondicionado de la empresa, pues el art. 18 del E.T., precepto al amparo del cual la demandada efectuó el registro, lo condiciona a que ello sea necesario para la protección del patrimonio empresarial y del de los demás trabajadores de la empresa".

En cambio, otros pronunciamientos niegan la virtualidad del artículo 18 ET en estas circunstancias. Asó lo hace la sentencia del TSJ de Madrid de 4 de diciembre de 2001: "debiendo rechazarse la pretensión de la parte actora, relativa a que el ordenador propiedad de la empresa y asignado el actor como berramienta de trabajo, no pudiera ser inspeccionado o comprobado por los responsables del departamento de sistemas informáticos de la demandada, sin incurrir en la infracción del art. 18 del ET, el cual solo establece la inviolabilidad de la personal del trabajador en sus taquillas y efectos particulares, cuando sean necesarios para la protección del patrimonio empresarial y de los demás trabajadores de la empresa, con las garantias que en el precepto legal indicado se establecen; siendo, por otra parte, claro que las garantías previstas en el citado art. 18 no pueden extenderse, en ningún caso, a la utilización de los ordenadores que la empresa asigne a su empleador, ya que tal útil de trabajo no puede considerarse como un efecto personal'. En idéntico sentido se pronuncian las sentencias de las salas de lo social de los TSJ de Cataluña de 5 de julio de 2000, que declara admisible una auditoría informática sin las garantías que éste reconoce precepto reconoce; y de Madrid de 13 de noviembre de 2001.

La discutida aplicación del artículo 18 ET, cuyo supuesto de hecho está en principio muy alejado del que nos ocupa, se explica por ser la única previsión sobre registros disponible en el Estatuto. También es una regulación que se ha aplicado de forma analógica a otras situaciones que no son estrictamente la taquilla y efectos personales: automóvil, mesa del trabajador, etc...; por eso puede entenderse que el artículo 18 ET contiene una regla general de protección de la intimidad del trabajador, sin que pueda interpretarse literalmente. La razón más directa, sin embargo, es que es muchas vèces là empresa la que lo alega para justificar el registro; el articulo 18 se lee así no como un mecanismo de garantía, sino como un título habilitante para éste ${ }^{7}$.

7. Aunque resulta indudable que se trata de una solución forzada, toda vez que entre la taquilla y el ordenador existen diferencias sustanciales. La más importante, que mientras que la taquilla es un. bien propiedad de la empresa, que se pone a disposición del trabajador para un uso privado, el ordenador se le atribuye para un uso estrictamente profesional; ademăs, en el caso dẹl ordenador: existen unas implicaciones, el secreto de las comunicaciones, que no existen en el asó de la" taquilla. 
Una segunda cuestión es la relativa a la vigencia del secreto de la correspondencia a estas comunicaciones privadas sobre medios de la empresa. No se aplica, para la STSJ de Cataluña de 5 de junio de 2002: "(...) el artículo 11.1 de la LOPJ señala que no surtirán efecto las pruebas obtenidas, directa o indirectamente, violentando los derechos o libertades fundamentales, pero en el presente caso no se ba vulnerado el derecho fundamental al secreto de las comunicaciones, ya que no nos encontramos en presencia de una correspondencia privada entre particulares cuyo secreto debe ser preservado, sino ante una utilización indebida de medios e instrumentos de la empresa para fines ajenos a los estrictamente laborales, pudiendo la empleadora ejercer un control sobre la forma de utilizar tales medios, que son de su propiedad, así como sobre la propia actividad laboral del trabajador". Tampoco para la STSJ de Andalucía de 25 de febrero de 2002: "no podemos olvidar que dicho ordenador, es un instrumento de trabajo propiedad de la empresa y que no debe ser utilizado para otros fines distintos y diferentes que la realización de la actividad laboral, por lo que no cabe bablar de documentos personales incorporados al mismo". En el mismo sentido la sentencia de la Sala de lo Social del Tribunal Superior de Justicia de Galicia de 4 de octubre de 2001 ha admitido el despido de un trabajador por uso indebido del correo electrónico, aceptando la legitimidad del acceso por la empresa a los contenidos de las direcciones de Internet que había venido utilizando.

Por el contrario sí se aplica para la SJS n ${ }^{\circ} 2$ de Barcelona de 16 de septiembre de 2002: "de abi que no pueda afirmarse que el art. 20.3 LET otorga un marco juridico suficiente para amparar las monitorizaciones de ordenadores a fin de obtener el conocimiento de los ocupadores de los correos electrónicos personales de sus trabajadores. Es obvio, en este sentido, que dicha norma no tiene rango de ley orgánica (ex art. $81.1 \mathrm{CE}$ ), sin que tampoco le sea postulable la peculiar delegación a ley ordinaria que establece el art. 2.2 de la Ley Ongánica 1/1982, atendiendo a que no desarrolla expresamente el secreto de comunicaciones. Dicha norma sí da amparo jurídico -como reiteradamente ba puesto de manifiesto la doctrina- la intromisión en el derecho a la intimidad de los trabajadores en los registros personales o en sus efectos particulares o en su armario (art. 18 LET), siempre y cuando este registro se baga con las garantias legalmente contempladas y sin que, por otro lado, como ha puesto de manifiesto la doctrina (entre otras, STSJ Castilla La Mancha 16.09.1999), sea posible el registro de bienes de la empresa -como la mesa de trabajo- sin cumplir dichas garantias. Asi, no tendría sentido que nuestro sistema juridico permitiera -con el respaldo de ley orgánica- una limitación del derecho fundamental a la intimidad con una serie de garantias, mientras que la posible afectación al secreto de las comunicaciones no ostente ningün régimen de tutela"s. En el caso DEUTSCHE

8. El TC ha dicho que sí, pero en otro contexto, penal y por lo tanto más restrictivo; en obiter dicta y de manera poco concluyente. 
BANK el orden laboral decía que no, y se planteo ante la jurisdicción penal, a primera vista con éxito al admitirse la denuncia. Sin embargo, no habrá sentencia penal al haber sido absueltos los directivos acusados por el trabajador tras haber llegado a un acuerdo económico.

En tercer lugar, es frecuente que los tribunales planteen la necesidad de garantizar una cierta proporcionalidad en el control, partiendo de la existencia de un conflicto entre derechos fundamentales de trabajador y empresario, y aplicando la doctrina general del TC en esta materia. Para la sentencia del TC de 10 de julio de 2000: "babria que preguntarse cuando tal registro es necesario para "la protección del patrimonio empresarial y del de los demás trabajadores de la empresa" (Art, 18 E.T.). En este sentido el registro debe cumplir el principio de proporcionalidad, en la medida que "sea susceptible de conseguir el objetivo propuesto (juicio de idoneidad); en segundo lugar, que no existe ninguna otra medida más moderada que evite utilizar la mâs lesiva al derecho fundamental de la intimidad (juicio de necesidad); $y$, por último, que de la misma deriven más beneficios o ventajas para el interés general que perjuicios sobre otros bienes o valores en conflicto (juicio de proporcionalidad en sentido estricto)". En idéntico sentido la sentencia del TSJ de Madrid de 31 de enero de 2002 declara la invalidez de las pruebas obtenidas como consecuencia de la monitorización en el ordenador del trabajador, por haber violado el derecho a la intimidad personal; el tribunal considera que la medida empresarial puede calificarse como idónea, pero no como necesaria, al existir aplicaciones informáticas útiles al fin propuesto sin entrar en el ordenador personal de los trabajadores. En la STSJ de Cataluña de 4 de diciembre de 2001 se valoró positivamente que la empresa instalara un programa de control del ordenador del trabajador que no llegaba a entrar en los archivos. En la sentencia del JS $\mathbf{n}^{\circ} 31$ de Madrid de 26 de abril de 2001 se afirma que "la decisión de monitorizar su puesto de trabajo vulneraria (...) su derecho a la libertad sindical (pues seria) un sacrificio totalmente desproporcionado de dicbo derecho a la libertad sindical en relación con la finalidad perseguida.

En cuarto lugar, en algún pronunciamiento se argumenta la existencia de una obligación de informar al trabajador sobre los controles a los que va a estar sometido, en la línea de lo que hacen ya algunas legislaciones europeas. Afirma en este sentido el JS $\mathrm{n}^{\circ} 30$ de Madrid que "en aquellos casos en los que el trabajador vaja a ser objeto de un control que exceda de los estándares normales o que interfiera en su intimidad, entendemos que estamos ante una condición esencial que debiera ser objeto de información". Una mayoría de los tribunales aceptan, sin embargo, la validez de controles no anunciados a los trabajadores o a sus representantes.

En quinto lugar, es frecuente que los tribunales exijan al empleador justificar los registros por razones objetivas previamente a su realización. En la sentencia del JS $n^{\circ} 31$ de Madrid de 26 de marzo de 2001 se argumenta que "la empresa 
demandada no ha demostrado eficazmente, como se reiteró arriba, que exista causa de justificación para la monitorización". En la del JS n 32 de Barcelona de 16 de septiembre de 2002 se tiene en cuenta el dato que no "se justificaran causas objetivas o subjetivas a estos efectos". En el asunto estudiado por la sentencia del TSJ de Cataluña de 4 de diciembre de 2001 -despido de un trabajador por uso compulsivo de los juegos del ordenador- la empresa instaló un programa que controlaba el uso del ordenador, y el tribunal lo aceptó porque la medida de control informático de la empresa era "justificada (ya que existian razonables sospechas de la comisión por parte del empleado de graves irregularidades en su puesto de trabajo).

En sexto lugar puede señalarse algún otro aspecto interesante del control del uso privado de las TIC en la empresa. Así, la monitorización injustificada de un trabajador representante sindical puede suponer una violación de la libertad sindical, como señala el JS $n^{\circ} 31$ de Madrid en sentencia de 26 de marzo de 2001, "la empresa demandada no ba demostrado eficazmente, como se reiteró arriba, que exista causa de justificación para la monitorización de los meses de enero y febrero, ya que no existia otra razón para proceder a la misma que la condición sindical y representativa del actor, así como la existencia de una situación conflictiva con la demandada". Por otro lado, una monitarización excesiva puede constituir una conducta de acoso, como afirma la sentencia del JS $n^{\circ} 32$ de Barcelona de 16 de septiembre de 2002.

En séptimo lugar, y en relación con las consecuencias de un uso considerado indebido, los tribunales aceptan por lo general la validez del despido por violación de la buena fe contractual. Aunque también se encuentra en la jurisprudencia analizada la idea de que es necesaria una cierta proporcionalidad en la reacción de la empresa ante esta conducta, sin que todo uso indebido pueda justificar por sí mismo un despido: así, la sentencia del TSJ de Madrid de 12 de junio de 2001 no encuentra causa de despido en un acceso a la red para fines personales pero con poca repercusión sobre la producción; en cambio, este mismo TSJ sí encuentra causa de despido cuando la actividad del trabajador tenía que ver con la seguridad del tráfico aéreo, según la sentencia de 13 de noviembre de 2001. Las causas aducidas por la empresa para justificar el despido suelen estar relacionadas con la clăusula general de "vulneración de la buena fe contractual", ante la falta de previsiones expresas en los convenios colectivos; otras veces se usan las menciones al "uso indebido de medios de la empresa", frecuente en los regímenes disciplinarios de éstos; en ocasiones, finalmente, la reacción de la empresa se produce mediante la aplicación de otros artículos del convenio, como por ejemplo los que sancionan la "perturbación del orden en la empresa".

A la hora de valorar la reacción de la empresa los tribunales suelen valorar la actuación anterior de ésta, apreciando en particular la existencia de una acepta- 
ción tácita de estas prácticas. En consecuencia, un entorno de tolerancia del uso privado impide que éste pueda considerarse ilícito laboral, como señala la sentencia del TSJ de Madrid de 16 de octubre de 1998, "....se alega por el actor que fue "cabeza de turco" con la que la empresa de modo ejemplarizante decidió combatir una práctica generalizada en la empresa. El motivo ha de rechazarse. La supuesta "práctica" no se desprende del relato histórico de la sentencia. No se puede alegar además tolerancia empresarial cuando la empresa ya había manifestado un año antes su intención de sancionar disciplinariamente las navegaciones irregulares en la red de Internet que efectuaron los trabajadores".

Estas notas perfilan con razonable precisión la postura de los tribunales respecto tanto de las consecuencias de un uso privado de las TIC en la empresa como de las posibilidades de control que se reconocen a ésta. Cabría terminar recordando que no nos encontramos en realidad ante una construcción jurisprudencial nueva, sino que se trata en líneas generales de la misma doctrina ya establecida hace tiempo respecto del uso irregular del teléfono de la empresa.

\subsubsection{Tratamiento en la negociación colectiva}

El acuerdo más avanzado hasta la fecha en el tratamiento de estas cuestiones lo es sin duda en España el Convenio Colectivo de Telefónica para los años 20002002, que ha centrado la atención de los operadores jurídicos y que se está tomando como referente para otros procesos de negociación. Los temas relacionados con las TIC son objeto de una regulación muy detallada, como consecuencia del hecho, mencionado en el propio texto del convenio, de que "ambas partes son conscientes de la creciente importancia que están adquiriendo las nuevas tecnologías en todos los procesos de comunicación (correo electrónico, utilización de Internet, etc.), tanto profesional como personal, de forma que gradualmente se está produciendo una sustitución paulatina de los medios de comunicación tradicionales por éstos. Dado que esta nueva situación puede producir efectos no deseados, por la posible utilización no adecuada de los mismos, ambas partes consideran conveniente fijar las reglas que deben regir la utilización de las berramientas y medios técnicos puestos a disposición de los trabajadores por el empresario". El convenio basa su regulación de estas materias en dos premisas fundamentales: "en primer lugar, el legitimo derecho del empresario de controlar el uso adecuado de las berramientas y medios técnicos que pone a disposición del trabajador para realizar su actividad y, por otra parte, debe salvaguardarse el derecbo a la intimidad del mismo".

La cláusula 12 dedica un apartado a la "utilización del correo electrónico e Internet por los empleados", donde se afirma que "los empleados podrán utilizar el correo electrónico, la dirección e-mail, la Red Corporativa e Internet con libertad y. 
en el sentido más amplio posible, para el desempeño de las actividades de su puesto de trabajo"'. Pero, se añade, "con carâcter general, los empleados de Telefónica de España, S.A.U. no podrán utilizar el correo electrónico, la Red Corporativa, ni Internet para fines particulares". Esta prohibición general de usos privados es desarrollada por el convenio, que delimita los usos considerados inadecuados de los medios de comunicación de la empresa: "en este sentido, bajo ningún concepto podrán los empleados utilizar estos medios para realizar envios masivos de mensajes, enviar mensajes con anexos de gran tamaño (capacidad), ni realizar cualquier tipo de envio sin relación alguna con el desempeño profesional, que interfiera las comunicaciones del resto de empleados o perturbe el normal funcionamiento de la Red Corporativa. Igualmente, no está permitido el envio de cadenas de mensajes electrónicos, la falsificación de mensajes de correo electrónico, el envio de mensajes o imágenes de material ofensivo, inapropiado o con contenidos discriminatorios por razones de género, edad, sexo, discapacidad, etc., aquellos que promuevan el acoso sexual, asi como la utilización de la red para juegos de azar, sorteos, subastas, descarga de vídeo, audio $u$ otros materiales no relacionados con la actividad profesional".

El convenio detalla igualmente las consecuencias de un uso indebido de los medios de comunicación de la empresa por sus trabajadores: "el incumplimiento de estas normas determinará la utilización por la empresa de las restricciones que considere oportuno en la utilización de estos medios y la aplicación del régimen disciplinario, en su caso". Esta enumeración de prácticas expresamente prohibidas tiene una utilidad muy significativa para el desarrollo de la relación de trabajo, pues permite modalizar las sanciones en caso de incumplimiento. Ya se ha visto que uno de los problemas que plantea la jurisprudencia española relativa al uso indebido del correo electrónico e Internet es precisamente la inadecuación de la solución que ofrece el Derecho tradicional, el despido por transgresión de la buena fe contractual; en Telefónica, y dado que el convenio distingue unas prácticas prohibidas especialmente odiosas o perniciosas, se puede restringir esta sanción extrema a las prácticas ejemplificadas, y aplicar otras más ligeras o livianas a los meros usos particulares de los medios informáticos de la empresa.

Igualmente interesantes resultan las garantías que se reconocen para los trabajadores en caso de que la empresa se plantee actuar contra éstos por esta causa: "cuando existan indicios de uso ilícito o abusivo por parte de un empleado, la em-

9. Con la salvaguardia de que "siempre que precisen realizar un uso de estos medios que exceda el habitual, envíos masivos o de especial complejidad, utilizarán los cauces adecuados, de acuerdo con su jefe inmediato, para no causar daños en el desarrollo normal de las comunicaciones y en el funcionamiento de la Red Corporativa". 
presa realizará las comprobaciones oportunas $y$, si fuera preciso, realizará una auditoría en el ordenador del empleado o en los sistemas que ofrecen el servicio, que se efectuará en borario laboral y en presencia de algún representante de los trabajadores o de la organización sindical que proceda, en caso de afiliación, si el empleado lo desea, con respeto a la dignidad e intimidad del empleado". Se reconoce así el derecho de la empresa de efectuar registros en el ordenador del trabajador, sin que éste pueda ser considerado como protegido por el secreto de las comunicaciones u otra garantía de privacidad; pero también se reconocen expresamente garantías de carácter procedimental, mediante la presencia de representantes colectivos del trabajador.

Existe ya un cierto número de convenios que se ocupan del uso privado de las TIC en la empresa, pero lo normal es que se limiten a recoger una prohibición de éste, tipificándolo como una infracción susceptible de sanción disciplinaria. En términos generales resulta más conveniente para los trabajadores una prohibición expresa del uso privado de las TIC que la falta de toda regla al respecto, puesto que en el primer caso la consecuencia suele ser la imposición de una sanción disciplinaria leve o grave, mientras que en el segundo puede llegar a producirse un despido por ruptura de la buena fe contractual.

\subsection{Uso sindical de LAS TIC EN LA EMPRESA}

Otro de los problemas recurrentes en la práctica laboral española es del uso de los medios TIC de la empresa por los representantes de sus trabajadores. Es conocida la importancia que el Derecho español otorga a la comunicación de éstos con sus representados, para lo que les garantiza una serie de derechos instrumentales en el ET y la LOLS. Pero estos derechos son muy específicos, y se refieren a los instrumentos de comunicación tradicionales, como el tablón de anunciós o el reparto de publicidad, lo que contrasta con una práctica cada vez más generalizada de utilización de la intranet de la empresa o administración pública, y con las grandes ventajas de los nuevos medios frente a los contemplados por el Estatuto. De nuevo nos encontramos con una normativa obsoleta, en cuya aplicación los tribunales laborales están jugando un papel muy destacado.

\subsubsection{Tratamiento en la jurisprudencia laboral}

En estos momentos el ruling case es el conocido como "caso BBVA", que ha tenido mucho impacto en los medios sindicales e internautas europeos, y que marca la solución legislativa a la cuestión del uso sindical de las TIC en la empresa. Se trata de un caso que no sólo tiene un valor intrínseco como precedente, sino que ofrece una doctrina que podrá ser compartida o no, pero que desde luego es clara u directa. Aunque se trató de un asunto con unas circunstancias muy particulares - 
se toleraba el uso por las secciones sindicales, pero se prohibió a partir de un problema técnico que generó un uso abusivo, existiendo además normas expresas de la empresa sobre el uso del correo electrónico- el modo en que se presentó la controversia, y la consiguiente resolución judicial, ofrecen una solución de aplicación a todo tipo de situaciones.

El primer pronunciamiento en este caso lo fue de la Sala de lo Social de la Audiencia Nacional, en sentencia de 6 de febrero de 2001. Este pronunciamiento supuso un efímero reconocimiento al uso de correo electrónico como herramienta sindical de comunicación, partiendo de la inadecuación del Derecho vigente para ordenar el uso de estos nuevos instrumentos. A pesar de ello, y en virtud de una interpretación evolutiva de éste, se concluyó declarando "el derecho del Sindicato accionante $y$ de sus Secciones Sindicales en las Empresas del Grupo BBVA, demandadas en estos autos, a transmitir noticias de interes sindical a sus afiliados y a los trabajadores en general a través del correo electrónico (E-mail)", con la salvaguardia de que ello debía hacerse "con la mesura y normalidad inocua con que lo venía realizando (...) hasta el momento en, que se emitió una cantidad masiva de mensajes que colapsó el servidor interno de la Empresa". Esta doctrina, dictada en relación con un sindicato, bien podía entenderse extrapolable a la representación unitaria en la empresa, dado que se trataba de actualizar unas previsiones legales sobre derechos de comunicación a los nuevos entornos tecnológicos; y en el Derecho vigente los derechos de uno y otro tipo de representación, la legal y la unitaria, están construidos de forma similar.

Esta doctrina resultó ser frágil, pues la sentencia del TS de 26 de noviembre de 2001 anuló la de la SAN, afirmando que "no bay norma juridica alguna que conceda al sindicato el derecho a utilizar los medios informáticos del Banco para realizar la comunicación con sus afiliados y las secciones sindicales". En consecuencia, "la utilización deberá ser expresamente consentida por la empresa". El artículo 8 de la LOLS reconoce el derecho de los afiliados a recibir la información que les remita su sindicato, pero "no establece que sea la empresa la que deba facilitar los medios materiales para llevarla a cabon. La conclusión final es que "la utilización del sistema que boy se niega podrá ser objeto de negociación colectiva o acuerdo de cualquier tipo, pero, mientras no se obtenga, la utilización deberá ser expresamente consentida por la demandada".

Esta doctrina, que no supone una base particularmente útil para actualizar el tratamiento laboral de las comunicaciones sindicales en la empresa, puede considerarse ya consolidada, aunque se tratara de una única sentencia, y en casación ordinaria. Así lo han expresado algunos magistrados de la Sala IV del TS en diversos foros. Y los términos en los que se expresa la sentencia son tan concretos que resulta difícil pensar que vuelva a plantearse la cuestión ante el TS. Sea como fuere, lo cierto es que ha calado ya a la doctrina judicial de los TSJ, que han seguido 
las indicaciones del Supremo en este punto, como hace la sentencia del TSJ de Asturias de 1 de marzo de 2002: "el objetivo de la administración de modernizar los servicios públicos acercando su funcionamiento a los ciudadanos y empresas pasa por asumir los medios técnicos y electrónicos informáticos y telemáticos que numerosas normas ya prevén de forma específica. Pero la utilización de la red de la empresa (en este caso Administración) por correo electrónico para transmitir información sindical, no ba sido ordenada legalmente. No existe norma, convenio o acuerdo que lo imponga y asi las cosas los criterios interpretativos que establece el art. $3 .^{\circ} 1$ del CC, no permiten reconocer en términos absolutos, con base en los artículos de la Constitución, y los invocados de la LOLS de 2 Ago. 1985, el derecho a utilizar el medio de correo electrónico a través del servicios de la empresa para el ejercicio de la actividad sindical en la misma o recibir la información que le remita su sindicato".

\subsubsection{Tratamiento en la negociación colectiva}

De nuevo ha sido la negociación colectiva la que se ha ocupado de superar las limitaciones legales para garantizar el derecho al uso sindical de las TIC en la empresa. Dado que este uso está bastante generalizado, son varios los convenios los que se ocupan expresamente de regularlo; en muchas ocasiones, por el contrario, son acuerdos de empresa los que reconocen y ordenan el recurso a estos soportes. Así, el ya citado Convenio Colectivo de Telefónica dedica su artículo 12.2 a la sutilización del correo electrónico por la representación unitaria de los trabajadores y por los sindicatos", recogiendo una regulación bastante completa que, entre otras cosas, incluye:

- Uso del Tablón Sindical: "Existe en la empresa un portal al que se accede por la Intranet de la Empresa, denominado e.domus, o portal del empleado. Uno de sus canales, el Tablón Sindical, está a disposición de los sindicatos que tengan representación en el Comité Intercentros; desde él se puede acceder directamente a las páginas Web de dichos sindicatos, donde publican y difunden las comunicaciones e informaciones que consideren. Los empleados pueden acceder al mismo desde los ordenadores que tienen en su puesto de trabajo".

- Comunicaciones masivas: "Para los temas de especial consideración, la empresa facilitará los medios oportunos para que los sindicatos más representativos a nivel estatal y el Comité Intercentros, individual o conjuntamente, publiquen la informaciön que consideren precisa (Negociación Colectiva, elecciones sindicales $y$. otros eventos singulares), por medio del servicio Infobuzón y todos los empleados reciban esta comunicación (...) La Empresa dotará de los mecanismos necesarios para que los sindicatos estatales más representativos puedan dirigir cöreos a sus. afiliados de forma global. 
- Dotación y uso del correo electrónico por parte de las secciones sindicales: "La Empresa facilitará acceso a la Red corporativa, al correo electrónico y a Internet a los sindicatos que tengan la consideración de más representativos a nivel estatal. También dotará de una dirección de correo electrónico a las secciones sindicales provinciales de dichos sindicatos, si éstos están representados en el Comité de Empresa Provincial correspondiente, procurando, si fuera necesario y en la medida de lo posible, la adecuación de los medios informáticos de que disponen.

- Consecuencias de un uso indebido de estas facilidades: "La utilización indebida de estas direcciones, el envio masivo de correo de forma injustificada asi como la cesión del uso de esta dirección a otra organización no autorizada, determinará la pérdida de esta facilidad tan pronto la Empresa tenga constancia de ello, $y$ en su caso, la aplicación del régimen disciplinario."

Es también una buena muestra de regulación convencional de estas cuestiones el Acuerdo Complementario al III convenio colectivo estatal de Empresas de Trabajo Temporal suscrito por la dirección de ADECCO ETT y la sección sindical de COMFIA en el año 2001, cuyo artículo 5.3 se dedica expresamente a los "Medios de Comunicación", disponiendo que "en orden a facilitar los derechos de información que legalmente tienen reconocidos los representantes de los trabajadores, las Secciones Sindicales que tengan reconocidos los derechos del articulo 10 de la L.O.L.S. y' sean firmantes del presente acuerdo dispondrán, cada una de ellas, de una página "Web" en la "Intranet de la Empresa para la distribución de propaganda y publicidad de carácter sindical y de cuyas actualizaciones se informará con la misma periodicidad y por los mismos medios mediante los cuales se informa de otras actualizaciones que se producen en la citada "Intranet". Se afirma también que "el correo electrónico es de exclusivo uso profesional. No obstante lo anterior, los Representantes de los Trabajadores podrán bacer uso del mismo para comunicarse entre si. Para cualquier otro uso ajeno a lo anteriormente expuesto, se requerirá el acuerdo previo con la Dirección de la Empresa."

El Convenio de Uniprex SA para los años 2000-2002 incluye en su artículo 53 entre las "garantías sindicales uel derecho a utilizar el correo electrónico sin restricciones para la comunicación entre los trabajadores, las secciones sindicales constituidas y los comités de empresa o delegados de personal. En este sentido se dotará al Comité Intercentros y a los diferentes Comités de Empresa y Delegados de personal de dirección de correo electrónico con la que se pueda garantizar el citado derecho. Igualmente se garantiza el derecho a utilizar la Intranet e Internet por los trabajadores para acceder a sitios web de carácter sindical y/o relacionados con sus derechos laborales y civiles, debiendo hacer un uso adecuado de estos medios.Los representantes de los trabajadores barán un uso adecuado del correo electrónico tratando de evitar comunicaciones que por su extensión pudieran entorpecer el normal desarrollo del trabajo" 
Más recientemente, y también con un notable eco en los medios interesados, se ha firmado un acuerdo en Barklays Bank, de mayo de 2002, que predica como objetivo el de "actualizar los canales de comunicación sindicales existentes en la Entidad y adaptar estos a las nuevas tecnologias de un modo eficaz y razonable. El acuerdo, muy breve y ceñido a su objeto, fija una serie de puntos:

- La Entidad dotará a la Sección Sindical Estatal de CC.OO. de Barclays Bank (en adelante S.S. de CC.OO.) con una cuenta de correo electrónico a fin de que pueda a través de este medio informar y comunicarse con los empleados.

- Será la S.S. de CC.OO. quien designe a los usuarios de su cuenta de correo electrónico.

- Las comunicaciones emitidas desde la cuenta de correo de la S.S. de CC.OO. podrán llevar el logo de su Federación de CC.OO., si aquella lo decidiese.

- La comunicación vía correo electrónico entre la S.S. de CC.OO. y los emipleados se hará necesariamente entre dicha cuenta y la cuenta de correo particular del empleado, puesta a su disposición por la Entidad.

- La S.S. de CC.OO. será responsable del contenido de sus envíos y del buen uso de la mencionada cuenta.

- La S.S. de CC.OO. se compromete a disminuir progresivamente los comunicados y hojas sindicales publicadas por los medios tradicionales en proporción a la implementación de los medios electrónicos.

Para concluir resulta pertinente una mención al Convenio Colectivo Interprovincial de Cajas de Ahorros para los años 2001-2002, cuyo interés está en su propia extensión como norma sectorial; además, este convenio puede adelantar lø que puede ser la solución convencional en el futuro, sobre todo en los sectores que no sean específicamente tecnológicos: reconocimiento del problema, y establecimiento de pautas a nivel sectorial, pero tratamiento directo a nivel de empresa. En concreto, su artículo 22 afirma que "las partes acuerdan que mientras no exista una regulación expresa, de carácter general, que afecte al uso y utilización de medios tecnológicos (Internet, Intranet y correo electrónico), las Cajas de Aborros, sometidas al ámbito funcional de este Convenio Colectivo, abrirán procesos de negociación, con los representantes legales de los empleados, tendentes al establecimiento de las medidas que permitan el uso por los sindicatos de estos mediós, sin que ello afecte al proceso normal de actividad y producción." 


\section{PROPUESTAS DE ADAPTACIÓN DEL DERECHO ESPAÑOL A LOS DERE- CHOS ON-LINE}

Como se ha visto, la situación del Derecho español en relación con los derechos on-line debe considerarse insuficiente. En el Derecho del trabajo vigente no se han encontrado bases suficientes como garantizar su reconocimiento. $Y$ tampoco la construcción de un Derecho jurisprudencial sobre la utilización de las TIC en la empresa ha servido en España para superar las limitaciones legales. De un lado, incluso cuando se pueden destilar los principios jurídicos que conforman a la fecha de hoy un "Derecho electrónico en la empresa" éstos resultan por lo general insuficientes para construir una regulación completa de los derechos on-line. De otro, porque en muchos casos las soluciones jurisprudenciales son inadecuadas, llevando a conclusiones injustas o ineficientes; lo que no debe sorprender, toda vez que los jueces han tenido que operar aplicando un marco normativo, el del Estatuto de los Trabajadores, altamente superado. El somero análisis que se ha hecho de los casos más notorios conocidos hasta la fecha es una buena muestra de ello. A todo lo que se unen las dificultades adicionales que supone operar con decisiones judiciales como única base jurídica en sistemas continentales como el nuestro, en el que los mecanismos de creación judicial del Derecho son poco directos y eficaces. El resultado es que en el momento actual la legislación vigente es más concausa del problema que solución.

Por todo ello se ha planteado la necesidad de superar la situación actual mediante la reforma del marco normativo regulador de las relaciones laborales en la empresa, y su sustitución por uno nuevo, capaz de acoger y ordenar las nuevas realidades tecnológicas. Desde distintas instancias han partido propuestas de actuación, habiéndose generado una corriente de opinión en la nueva forma de operar de los movimientos sociales del siglo XXI: por medio de listas de correo, páginas web, chats y otros medios tecnológicos sujetos como partidos políticos, sindicatos, asociaciones de internautas o grupos de investigación están ofreciendo propuestas en esta dirección.

Fruto de esta línea de actuación aparecen las primeras propuestas. En concreto, el Grupo Parlamentario Mixto, a iniciativa del Senador de Izquierda Unida por la Comunidad Autónoma de Les Illes Balears, Manuel CAMARA FERNÁNDEZ, presentó el 17 de octubre de 2000 una moción para su debate en el Pleno del Senado que en su parte dispositiva indicaba lo siguiente:

El Senado insta al Gobierno a que ponga en marcha las medidas necesarias para considerar el correo electrónico e Internet como instrumentos de comunicación e información de los trabajadores y de los sindicatos en el seno de la empresa, incluyendo el derecho al uso de estos soportes telemáticos en la labor informativa sindical en el propio Estatuto de los Trabajadores, en el que se incluyan entre otras las siguientes cuestiones: 
1. El derecho de cada trabajador a un buzón personal de correo electrónico en la empresa.

2. El derecho de los smdicatos a comunicarse con los trabajadores a través de un buzón sindical de libre acceso en las empresas.

3. Libre acceso de los representantes sindicales al correo electrónico e Internet en la empresa para el desarrollo de su actividad sindical.

4. Libre acceso de los trabajadores al correo electrónico y a las páginas de Internet sindicales para obtener información laboral en la empresa.

5. Que las empresas respeten la inviolabilidad de las comunicaciones a través de Internet.

Durante el debate de esta moción en el Senado, se llegó a un cierto nivel de acuerdo que se tradujo el 28 de noviembre de 2000 con la aprobación por unanimidad el siguiente texto:

El Senado insta al Gobierno a que en el plazo más breve posible estudie la forma de poner en marcha las medidas necesarias para considerar el correo electrónico en Internet como instrumentos de comunicación e información de los trabajadores con sus representantes sindicales y viceversa, siempre que la actividad y características generales de las empresas lo permitan, facilitando el acceso de los trabajadores y sus representantes sindicales al correo electrónico de Internet de la empresa con garantía de inviolabilidad de las comunicaciones conforme al marco legal vigente.

La falta de desarrollo de esta moción motivó la presentación en mayo de 2001 mayo de una nueva iniciativa mucho más concreta en el Senado, en esta ocasión en forma de Proposición de Ley, por parte del mismo Senador CÁMARA Esta propuesta fue rechazada, pero sin duda merece nuestra atención por su interés.

La propuesta incluye, en primer lugar, una modificación del Estatuto de los Trabajadores y de la Ley Orgánica de Libertad Sindical para incluir expresamente el uso de las nuevas tecnologías dentro de las empresas y que éstas puedan ser utilizadas como instrumentos de transmisión de información entre los trabajadores y sus representantes. Para ello se propone la modificación del artículo 81 del Real Decreto Legislativo 1/1995 de 24 de marzo, texto refundido del Estatuto de los trabajadores. En éste, como es sabido, se reconoce el derecho de los órganos de representación unitaria a disponer de un local y tablón de anunciós; con la propuesta se le añadiría un inciso al final de su redacción actual con el siguiente ténor literal: "asimismo cuando en las empresas estén suficientemente implantadias las Nuevas Tecnologias de la Información, se les facilitará un lugar en la intrànet de la empresa y los medios técnicos necesarios para garantizar la comunićáción electrónica entre trabajadores y representación legal.. 